\title{
GPCR interactions in space and time
}

A proximity labeling technique yields new insights into GPCR signaling.

G-protein-coupled receptors, or GPCRs, are a large family of transmembrane proteins that have crucial biological signaling roles and represent the most important class of drug targets. A great deal of attention has been focused on such receptors. However, many questions about GPCR signaling remain-particularly about what proteins the receptors engage, and when.

Techniques such as affinity purification coupled with mass spectrometry enable the identification of protein interactions, but they do not capture kinetic information. Recent work from two separate groups now shows that a rapid proximity labeling approach can be applied to monitor GPCR interactions in both space and time.

University of California, San Francisco (UCSF) researchers Nevan Krogan and Mark von Zastrow brought together their complementary expertise in proteomics technology and GPCR biology, respectively, to investigate protein interactions that have been intractable to traditional techniques. They selected a tool for proximity-based biotinylation called APEX (engineered ascorbic acid peroxidase), originally developed in the lab of Alice Ting (now of Stanford University). This approach has been used to successfully map organelle proteomes with high precision by targeting APEX to the organelle of interest, incubating cells with biotin-phenol and adding hydrogen peroxide to trigger the biotinylation reaction. Proteins in close proximity to APEX are rapidly biotinylated; such proteins can be captured by streptavidin-based affinity chromatography and identified by massspectrometry-based proteomics methods.

APEX has very fast labeling kinetics, which is important for capturing transient GPCR signaling interactions. However, while its broad, unspecific labeling capability is an asset for defining organelle proteomes, the UCSF group questioned whether APEX had the necessary spatial resolution to define specific protein interaction networks. By targeting APEX to different organelles and identifying spatial reference proteins, they could locate the GPCR and also normalize for background biotinylation.

First authors Braden Lobingier and Ruth Hüttenhain confirmed that they could capture known interactions of beta- 2 adrenergic receptor ( $\beta 2 \mathrm{AR})$, a well-studied GPCR. They then moved to the less studied delta opioid receptor (DOR), exposing cells to an opioid agonist for varying time periods, which led them to identify 48 interacting proteins. Using a targeted proteomics approach for quantification of protein abundance, they validated 29 of these interactions as being specific, classifying them as being biotinylated at an early, middle or late phase following agonist binding. They followed up on two ubiquitin-linked proteins that were engaged at a late phase, suggesting their novel roles as DOR degraders.

"GPCRs are incredibly well studied, yet we identified several new factors involved at different stages of GPCR functioning after stimulation with a ligand," Krogan notes. With some development, he says, the method could likely be applied to cytoplasmic as well as membrane proteins, though he is most interested in applying APEX to map the signaling networks of other GPCRs, such as the serotonin and dopamine receptors.

The time resolution offered by APEX also attracted Harvard Medical School (HMS) researcher Andrew Kruse. To gain new insights into GPCR signaling, he needed an approach that could monitor rapid receptor activation and internalization upon treatment with a ligand, all in a native-like setting.

Kruse worked with HMS colleague and proteomics expert Steven Gygi to quantify proteins labeled by an APEX-tagged GPCR when exposed to a ligand for different timepoints. Along with first authors, Jaeho Paek and Marian Kalocsay, they contended with the broad labeling of APEX

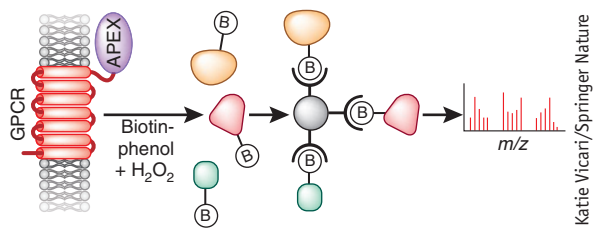

Proximity biotinylation by APEX captures GPCR-protein interactions identified using mass spectrometry.

by using an isobaric tagging technique for accurate quantification by mass spectrometry; through careful statistical analysis of their data, they identified enriched proteins standing out from the background.

In studying $\beta 2 \mathrm{AR}$, a class A GPCR representative, and the angiotensin II type 1 receptor (AT1R), a class B receptor, they observed waves of proteins engaged by the GPCRs at different times, as the GPCRs were activated and internalized. Their results recapitulated what was known about receptor dynamics, but the group also found something novel: LMBRD2, a previously uncharacterized protein that appears to play a role in the endocytosis and recycling of both $\beta 2 \mathrm{AR}$ and AT1R.

Kruse believes that APEX will be broadly applicable. "Many biologically interesting proteins don't belong to large families like the GPCRs," he points out. "Proximity labeling with isobaric tagging offers a new approach to interrogate their signaling without requiring much prior knowledge."

He notes that both studies, which the groups submitted in parallel to Cell, involve the $\beta 2 \mathrm{AR}$ receptor. "The fact that [APEX] gives such similar results in different labs 3,000 miles apart is a remarkable validation of its utility and reproducibility," he says.

\section{Allison Doerr}

\section{RESEARCH PAPERS}

Lobingier, B.T. et al. An approach to spatiotemporally resolve protein interaction networks in living cells. Cell 169, 350-360 (2017).

Paek, J. et al. Multidimensional tracking of GPCR signaling via peroxidase-catalyzed proximity labeling. Cell 169, 338-349 (2017). 